\title{
Trace inequalities for positive semidefinite matrices with centrosymmetric structure
}

\author{
Di Zhao ${ }^{1}$, Hongyi Li ${ }^{1 *}$ and Zhiguo Gong ${ }^{2}$
}

\author{
* Correspondence: \\ Hongyili_buaa@163.com \\ ${ }^{1}$ LMIB, School of Mathematics and \\ System Science, Beihang University, \\ Beijing, China \\ Full list of author information is \\ available at the end of the article
}

\section{Abstract}

In this article, we present some results on the Hadamard product of positive semidefinite matrices with centrosymmetric structure. Based on these results, several trace inequalities on positive semidefinite centrosymmetric matrices are obtained.

\section{Introduction and preliminaries}

We will use the following notation. Let $\mathbb{C}^{n \times n}$ and $\mathbb{R}^{n \times n}$ be the space of $n \times n$ complex and real matrices, respectively. The identity matrix in $\mathbb{C}^{n \times n}$ is denoted by $I=I_{n}$. Let $A^{T}, \bar{A}, A^{H}$, and $\operatorname{tr}(A)$ denote the transpose, the conjugate, the conjugate transpose, and the trace of a matrix $A$, respectively. Let $\operatorname{Re}(a)$ represent the real part of $a$. The Frobenius inner product $\langle\cdot, \cdot\rangle_{F}$ in $\mathbb{C}^{m \times n}$ over the complex field is defined as follows: $<A, B>_{F}=\operatorname{Re}\left(\operatorname{tr}\left(B^{H} A\right)\right)$, for $A, B \in \mathbb{C}^{m \times n}$, i.e., $<A, B>$ is the real part of the trace of $B^{H} A$. The induced matrix norm is $\|A\|_{F}=\sqrt{<A, A>_{F}}=\sqrt{\operatorname{Re}\left(\operatorname{tr}\left(A^{H} A\right)\right)}=\sqrt{\operatorname{tr}\left(A^{H} A\right)}$, which is called the Frobenius (Euclidean) norm.

A matrix $A \in \mathbb{C}^{n \times n}$ is Hermitian if $A^{H}=A$, and $A$ is called positive semidefinite, written as $A \geq 0$ (see [1, p. 159]), if

$$
x^{H} A x \geq 0, \quad \forall x \in \mathbb{C}^{n} .
$$

$A$ is further called positive definite, symbolized $A>0$, if the strict inequality in (1.1) holds for all non-zero $x \in \mathbb{C}^{n}$. An equivalent condition for $A \in \mathbb{C}^{n}$ to be positive definite is that $A$ is Hermitian and all eigenvalues of $A$ are positive.

Let $A$ and $B$ be two Hermitian matrices of the same size. If $A-B$ is positive semidefinite, we write

$$
A \geq B .
$$

Next, we introduce some basic definitions and lemmas.

Definition 1.1 (see [1]). Let $A$ be a square complex matrix partitioned as

$$
A=\left(\begin{array}{ll}
A_{11} & A_{12} \\
A_{21} & A_{22}
\end{array}\right)
$$

where $A_{11}$ is a square submatrix of $A$. If $A_{11}$ is nonsingular, we call $\tilde{A}_{11}=A_{22}-A_{21} A_{11}^{-1} A_{12}$ the Schur complement of $A_{11}$ in $A$.

Note. If $A$ is a positive definite matrix, then $A_{11}$ is nonsingular and $A_{22} \geq \tilde{A}_{11} \geq 0$.

(c) 2012 Zhao et al; licensee Springer. This is an Open Access article distributed under the terms of the Creative Commons Attribution License (http://creativecommons.org/licenses/by/2.0), which permits unrestricted use, distribution, and reproduction in any medium, provided the original work is properly cited. 
Definition 1.2 (see [2]). $A=\left(a_{i j}\right) \in \mathbb{C}^{n \times n}$ is called a centrosymmetric matrix, if

$$
a_{i j}=a_{n-i+1, n-j+1}, 1 \leq i \leq n, 1 \leq j \leq n, \text { or } J_{n} A J_{n}=A,
$$

where $J_{n}=\left(e_{n}, e_{n-1}, \ldots, e_{1}\right), e_{i}$ denotes the unit vector with the ith entry 1.

If a matrix is both positive semidefinite and centrosymmetric, we call this matrix positive semidefinite centrosymmetric.

Using the partition of matrix, the central symmetric character of a square centrosymmetric matrix can be described as follows [2]:

Lemma 1.1 (see [2]). Let $A=\left(a_{i j}\right) \in \mathbb{C}^{n \times n}(n=2 m)$ be centrosymmetric. Then, $A$ has the following form,

$$
A=\left(\begin{array}{ll}
B J_{m} C J_{m} \\
C J_{m} B J_{m}
\end{array}\right), \quad \text { and } P^{T} A P=\left(\begin{array}{cc}
B-J_{m} C & 0 \\
0 & B+J_{m} C
\end{array}\right),
$$

where $B \in \mathbb{C}^{m \times m}, C \in \mathbb{C}^{m \times m}, P=\frac{1}{\sqrt{2}}\left(\begin{array}{cc}I_{m} & I_{m} \\ -J_{m} & J_{m}\end{array}\right)$.

Note. In this article, we mainly discuss the case $n=2 m$. For $n$ is odd, i.e., $n=2 m+$ 1 , similar results can be obtained by taking similar steps.

Recently, in [3], Ulukök and Türkmen proved some matrix trace inequalities for positive semidefinite matrices:

Lemma 1.2 (see [3]). Let $A, B \in \mathbb{C}^{n \times n}$. Then,

$$
\left\|(A \circ B)^{m}\right\|_{F}^{2} \leq\left\|\left(A^{H} A\right)^{m}\right\|_{F} \cdot\left\|\left(B^{H} B\right)^{m}\right\|_{F}
$$

where $m$ is an positive integer, $A \circ B$ stands for the Hadamard product of $A$ and $B$.

Note. Particularly, if $A$ and $B$ in Lemma 1.2 are both semidefinite matrices, then $\left\|(A \circ B)^{m}\right\|_{F}^{2} \leq\left\|A^{2 m}\right\|_{F} \cdot\left\|B^{2 m}\right\|_{F}$.

Lemma 1.3 (see [3]). Let $A_{i} \in \mathbb{C}^{n \times n},(i=1,2, \ldots, k)$ be semidefinite matrices. Then, for positive real numbers $s, m, t$

$$
\left(\sum_{i=1}^{k}\left\|A_{i}^{((s+t) / 2) m}\right\|_{F}^{2}\right) \leq\left(\sum_{i=1}^{k}\left\|A_{i}^{s m}\right\|_{F}^{2}\right)\left(\sum_{i=1}^{k}\left\|A_{i}^{t m}\right\|_{F}^{2}\right)
$$

Lemma 1.4 (see [3]). Let $A=\left(\begin{array}{ll}A_{11} & A_{12} \\ A_{21} & A_{22}\end{array}\right) \geq 0, B=\left(\begin{array}{ll}B_{11} & B_{12} \\ B_{21} & B_{22}\end{array}\right) \geq 0$. Then,

$$
\operatorname{tr}\left[\left(\tilde{A}_{22}\right)^{1 / 2} B_{11}^{1 / 2}\right]^{2 m}+\operatorname{tr}\left[\left(A_{22}\right)^{1 / 2} \tilde{B}_{11}^{1 / 2}\right]^{2 m} \leq \operatorname{tr}(A B)^{m} \leq \operatorname{tr}\left(A^{m} B^{m}\right),
$$

where $m$ is an integer.

\section{Main results}

Lemma 2.1. Let $A=\left(a_{i j}\right)_{n \times n}, B=\left(b_{i j}\right)_{n \times n}(n=2 m)$ be two centrosymmetric matrices with the following form:

$$
A=\left(\begin{array}{l}
A_{1} J_{m} A_{2} J_{m} \\
A_{2} J_{m} A_{1} J_{m}
\end{array}\right), \quad B=\left(\begin{array}{l}
B_{1} J_{m} B_{2} J_{m} \\
B_{2} J_{m} B_{1} J_{m}
\end{array}\right), A_{1}, A_{2}, B_{1}, B_{2} \in \mathbb{C}^{m \times m}
$$

Then, $A \circ B$ is a centrosymmetric matrix. 
Proof. By the definition of Hadamard product,

$$
A \circ B=\left(\begin{array}{l}
A_{1} J_{m} A_{2} J_{m} \\
A_{2} J_{m} A_{1} J_{m}
\end{array}\right) \circ\left(\begin{array}{l}
B_{1} J_{m} B_{2} J_{m} \\
B_{2} J_{m} B_{1} J_{m}
\end{array}\right)=\left(\begin{array}{l}
A_{1} \circ B_{1}\left(J_{m} A_{2} J_{m}\right) \circ\left(J_{m} B_{2} J_{m}\right) \\
A_{2} \circ B_{2}\left(J_{m} A_{1} J_{m}\right) \circ\left(J_{m} B_{1} J_{m}\right)
\end{array}\right)
$$

We shall prove the following

$$
\left\{\begin{array}{l}
\left(J_{m} A_{1} J_{m}\right) \circ\left(J_{m} B_{1} J_{m}\right)=J_{m}\left(A_{1} \circ B_{1}\right) J_{m}, \\
\left(J_{m} A_{2} J_{m}\right) \circ\left(J_{m} B_{2} J_{m}\right)=J_{m}\left(A_{1} \circ B_{2}\right) J_{m} .
\end{array}\right.
$$

From (2.1),

$$
A_{1} \circ B_{1}=\left(\begin{array}{lll}
a_{11} & \cdots & a_{1 m} \\
\vdots & \ddots & \vdots \\
a_{m 1} & \cdots & a_{m m}
\end{array}\right) \circ\left(\begin{array}{lll}
b_{11} & \cdots & b_{1 m} \\
\vdots & \ddots & \vdots \\
b_{m 1} & \cdots & b_{m m}
\end{array}\right)=\left(\begin{array}{lll}
a_{11} b_{11} & \cdots & a_{1 m} b_{1 m} \\
\vdots & \ddots & \vdots \\
a_{m 1} b_{m 1} & \cdots & a_{m m} b_{m m}
\end{array}\right),
$$

and

$$
J_{m}\left(A_{1} \circ B_{1}\right) J_{m}=\left(\begin{array}{lll}
a_{m m} b_{m m} & \cdots & a_{m 1} b_{m 1} \\
\vdots & \ddots & \vdots \\
a_{1 m} b_{1 m} & \cdots & a_{11} b_{11}
\end{array}\right) .
$$

Since

$$
J_{m} A_{1} J_{m}=\left(\begin{array}{lll}
a_{m m} & \cdots & a_{m 1} \\
\vdots & \ddots & \vdots \\
a_{1 m} & \cdots & a_{11}
\end{array}\right), \text { and } J_{m} B_{1} J_{m}=\left(\begin{array}{lll}
b_{m m} & \cdots & b_{m 1} \\
\vdots & \ddots & \vdots \\
b_{1 m} & \cdots & b_{11}
\end{array}\right),
$$

we have the following

$$
\left(J_{m} A_{1} J_{m}\right) \circ\left(J_{m} B_{1} J_{m}\right)=\left(\begin{array}{lll}
a_{m m} b_{m m} & \cdots & a_{m 1} b_{m 1} \\
\vdots & \ddots & \vdots \\
a_{1 m} b_{1 m} & \cdots & a_{11} b_{11}
\end{array}\right) .
$$

From (2.5) and (2.7), it is clear that

$$
\left(J_{m} A_{1} J_{m}\right) \circ\left(J_{m} B_{1} J_{m}\right)=J_{m}\left(A_{1} \circ B_{1}\right) J_{m} .
$$

Similarly, we can prove that

$$
\left(J_{m} A_{2} J_{m}\right) \circ\left(J_{m} B_{2} J_{m}\right)=J_{m}\left(A_{2} \circ B_{2}\right) J_{m} .
$$

From (2.8) and (2.9), we can see that (2.3) holds. By Lemma 1.1, $A \circ B$ is a centrosymmetric matrix.

Lemma 2.2 (see [4]) Let $A=\left(a_{i j}\right)_{n \times n}(n=2 m)$ a positive semidefinite centrosymmetric matrix with the following form

$$
A=\left(\begin{array}{l}
B J_{m} C J_{m} \\
C J_{m} B J_{m}
\end{array}\right), B, C \in \mathbb{C}^{m \times m}
$$

Let $M=B-J_{m} C$ and $N=B+J_{m} C$. Then, $M, N$ are positive semidefinite matrices.

Theorem 2.1 Let $A, B \in \mathbb{C}^{n \times n}(n=2 m)$ be two positive semidefinite centrosymmetric matrices with the same form as in (2.1). Let

$$
M_{A}=A_{1}-J_{m} A_{2}, \quad N_{A}=A_{1}+J_{m} A_{2}, \quad M_{B}=B_{1}-J_{m} B_{2}, \quad N_{B}=B_{1}+J_{m} B_{2},
$$


and

$$
X=A_{1} \circ B_{1}-J_{m}\left(A_{2} \circ B_{2}\right), \quad Y=A_{1} \circ B_{1}+J_{m}\left(A_{2} \circ B_{2}\right) .
$$

Then, the following inequality holds:

$$
\left\|X^{m}\right\|_{F}^{2}+\left\|Y^{m}\right\|_{F}^{2} \leq \sqrt{\left(\left\|M_{A}^{2 m}\right\|_{F}^{2}+\left\|N_{A}^{2 m}\right\|_{F}^{2}\right) \cdot\left(\left\|M_{B}^{2 m}\right\|_{F}^{2}+\left\|N_{B}^{2 m}\right\|_{F}^{2}\right)} .
$$

Proof. Since $A, B$ are centrosymmetric matrices, by Lemma $2.1, A \circ B$ is centrosymmetric and

$$
A \circ B=\left(\begin{array}{l}
A_{1} \circ B_{1}\left(J_{m} A_{2} J_{m}\right) \circ\left(J_{m} B_{2} J_{m}\right) \\
A_{2} \circ B_{2}\left(J_{m} A_{1} J_{m}\right) \circ\left(J_{m} B_{1} J_{m}\right)
\end{array}\right) .
$$

From Lemma 1.1,

$$
P^{T}(A \circ B) P=\left(\begin{array}{cc}
A_{1} \circ B_{1}-J_{m}\left(A_{2} \circ B_{2}\right) & 0 \\
0 & A_{1} \circ B_{1}-J_{m}\left(A_{2} \circ B_{2}\right)
\end{array}\right)=\left(\begin{array}{cc}
X & 0 \\
0 & Y
\end{array}\right) .
$$

$P$ is orthogonal, then

$$
P^{T}(A \circ B)^{m} P=\left(\begin{array}{cc}
X^{m} & 0 \\
0 & Y^{m}
\end{array}\right),
$$

and

$$
\left\|(A \circ B)^{m}\right\|_{F}^{2}=\left\|P^{T}(A \circ B)^{m} P\right\|_{F}^{2}=\left\|\left(\begin{array}{cc}
X^{m} & 0 \\
0 & Y^{m}
\end{array}\right)\right\|_{F}^{2}=\left\|X^{m}\right\|_{F}^{2}+\left\|Y^{m}\right\|_{F}^{2} .
$$

Similarly from Lemma 1.1,

$$
\begin{aligned}
& P^{T} A P=\left(\begin{array}{cc}
A_{1}-J_{1} A_{2} & 0 \\
0 & A_{1}+J_{1} A_{2}
\end{array}\right)=\left(\begin{array}{cc}
M_{A} & 0 \\
0 & N_{A}
\end{array}\right), \\
& P^{T} B P=\left(\begin{array}{cc}
B_{1}-J_{1} B_{2} & 0 \\
0 & B_{1}+J_{1} B_{2}
\end{array}\right)=\left(\begin{array}{cc}
M_{B} & 0 \\
0 & N_{B}
\end{array}\right) .
\end{aligned}
$$

Then,

$$
P^{T} A^{2 m} P=\left(\begin{array}{cc}
M_{A}^{2 m} & 0 \\
0 & N_{A}^{2 m}
\end{array}\right), P^{T} B^{2 m} P=\left(\begin{array}{cc}
M_{B}^{2 m} & 0 \\
0 & N_{B}^{2 m}
\end{array}\right),
$$

and

$$
\begin{array}{r}
\left\|A^{2 m}\right\|_{F} \cdot\left\|B^{2 m}\right\|_{F}=\left\|\left(\begin{array}{cc}
M_{A}^{2 m} & 0 \\
0 & N_{A}^{2 m}
\end{array}\right)\right\|_{F} \cdot\left\|\left(\begin{array}{cc}
M_{B}^{2 m} & 0 \\
0 & N_{B}^{2 m}
\end{array}\right)\right\|_{F} \\
=\sqrt{\left(\left\|M_{A}^{2 m}\right\|_{F}^{2}+\left\|N_{A}^{2 m}\right\|_{F}^{2}\right) \cdot\left(\left\|M_{B}^{2 m}\right\|_{F}^{2}+\left\|N_{B}^{2 m}\right\|_{F}^{2}\right) .}
\end{array}
$$

Since both $A$ and $B$ are positive semidefinite matrices, by Lemma 1.2

$$
\|(A \circ B)\|_{F}^{2} \leq\left\|A^{2 m}\right\|_{F} \cdot\left\|B^{2 m}\right\|_{F} .
$$

Thus,

$$
\left\|X^{m}\right\|_{F}^{2}+\left\|Y^{m}\right\|_{F}^{2} \leq \sqrt{\left(\left\|M_{A}^{2 m}\right\|_{F}^{2}+\left\|N_{A}^{2 m}\right\|_{F}^{2}\right) \cdot\left(\left\|M_{B}^{2 m}\right\|_{F}^{2}+\left\|N_{B}^{2 m}\right\|_{F}^{2}\right)} .
$$


Theorem 2.2. Let $A \in \mathbb{C}^{n \times n}(n=2 m)$ be postive semidefinite centroysymmetric with the form:

$$
A=\left(\begin{array}{l}
B J_{m} C J_{m} \\
C J_{m} B J_{m}
\end{array}\right), \quad B, C \in \mathbb{C}^{m \times m} .
$$

Let $M=B-J_{m} C, N=B+J_{m} C$. Then, for positive integers $s, m$, $t$, the following two equalities hold

$$
\left\|A^{((s+t) / 2) m}\right\|_{F}^{2}=\left\|M^{((s+t) / 2) m}\right\|_{F}^{2}+\left\|N^{((s+t) / 2) m}\right\|_{F}^{2},
$$

and

$$
\left\|M^{((s+t) / 2) m}\right\|_{F}^{2}+\left\|N^{((s+t) / 2) m}\right\|_{F}^{2} \leq\left(\left\|M^{s m}\right\|_{F}^{2}+\left\|N^{s m}\right\|_{F}^{2}\right) \cdot\left(\left\|M^{t m}\right\|_{F}^{2}+\left\|N^{t m}\right\|_{F}^{2}\right) .
$$

Proof. By Lemma 1.1,

$$
P^{T} A P=\left(\begin{array}{cc}
B-J_{m} C & 0 \\
0 & B+J_{m} C
\end{array}\right)=\left(\begin{array}{cc}
M & 0 \\
0 & N
\end{array}\right) .
$$

Since $A$ is positive semidefinite, we have

$$
P^{T} A^{((s+t) / 2) m} P=\left(\begin{array}{cc}
M^{((s+t) / 2) m} & 0 \\
0 & N^{((s+t) / 2) m}
\end{array}\right) .
$$

Thus,

$$
\begin{aligned}
\left\|A^{((s+t) / 2) m}\right\|_{F}^{2}=\left\|P^{T} A^{((s+t) / 2) m}\right\|_{F}^{2} & =\left\|\left(\begin{array}{cc}
M^{((s+t) / 2) m} & 0 \\
0 & N^{((s+t) / 2) m}
\end{array}\right)\right\|_{F}^{2} \\
& =\left\|M^{((s+t) / 2) m}\right\|_{F}^{2}+\left\|N^{((s+t) / 2) m}\right\|_{F}^{2} .
\end{aligned}
$$

From Lemma 2.2, $M$ and $N$ are positive semidefinite. Combining Lemma 1.3, we have

$$
\left\|M^{((s+t) / 2) m}\right\|_{F}^{2}+\left\|N^{((s+t) / 2) m}\right\|_{F}^{2} \leq\left(\left\|M^{s m}\right\|_{F}^{2}+\left\|N^{s m}\right\|_{F}^{2}\right) \cdot\left(\left\|M^{t m}\right\|_{F}^{2}+\left\|N^{t m}\right\|_{F}^{2}\right) .
$$

Theorem 2.3 Let $A, B \in \mathbb{C}^{n \times n}(n=2 m)$ are positive semidefinite centrosymmetric matrices with the same form as in (2.1). Let

$$
M_{A}=A_{1}-J_{m} A_{2}, \quad N_{A}=A_{1}+J_{m} A_{2}, \quad M_{B}=B_{1}-J_{m} B_{2}, \quad N_{B}=B_{1}+J_{m} B_{2} .
$$

Then, the following inequality holds

$$
\operatorname{tr}\left(M_{A}^{1 / 2} M_{B}^{1 / 2}\right)^{2 m}+\operatorname{tr}\left(N_{A}^{1 / 2} N_{B}^{1 / 2}\right)^{2 m} \leq \operatorname{tr}(A B)^{m} \leq \operatorname{tr}\left(A^{m} B^{m}\right) .
$$

Proof. From Lemma 1.1, there exists an orthogonal matrix $P$ such that

$$
\begin{aligned}
& P^{T} A P=\left(\begin{array}{cc}
A_{1}-J_{m} A_{2} & 0 \\
0 & A_{1}+J_{m} A_{2}
\end{array}\right)=\left(\begin{array}{cc}
M_{A} & 0 \\
0 & N_{A}
\end{array}\right), \\
& P^{T} B P=\left(\begin{array}{cc}
B_{1}-J_{m} B_{2} & 0 \\
0 & B_{1}+J_{m} B_{2}
\end{array}\right)=\left(\begin{array}{cc}
M_{B} & 0 \\
0 & N_{B}
\end{array}\right) .
\end{aligned}
$$


According to Definition 1.1,

$$
\tilde{N}_{A}=M_{A}-0 \cdot N_{A}^{-1} \cdot 0=M_{A}, \tilde{M}_{B}=N_{B}-0 \cdot M_{B}^{-1} \cdot 0=N_{B} .
$$

From Lemma 1.4,

$$
\operatorname{tr}\left(M_{A}^{1 / 2} M_{B}^{1 / 2}\right)^{2 m}+\operatorname{tr}\left(N_{A}^{1 / 2} N_{B}^{1 / 2}\right)^{2 m} \leq \operatorname{tr}\left(P^{T} A P P^{T} B P\right)^{m}=\operatorname{tr}\left(P^{T} A B P\right)^{m} .
$$

Since $P$ is orthogonal, we have $\operatorname{tr}\left(P^{T} A B P\right)^{m}=\operatorname{tr}(A B)^{m}$. By Lemma 1.4, the following holds

$$
\operatorname{tr}\left(M_{A}^{1 / 2} M_{B}^{1 / 2}\right)^{2 m}+\operatorname{tr}\left(N_{A}^{1 / 2} N_{B}^{1 / 2}\right)^{2 m} \leq \operatorname{tr}(A B)^{m} \leq \operatorname{tr}\left(A^{m} B^{m}\right) .
$$

\section{Acknowledgements}

We would like to thank the reviewers for providing valuable comments and suggestions to improve the manuscript. This study was supported by the National Natural Science Foundation of China (No. 60831001).

\section{Author details}

${ }^{1}$ LMIB, School of Mathematics and System Science, Beihang University, Beijing, China ${ }^{2}$ Faculty of Science and

Technology, University of Macau, C. Postal 3001 Macau, China

\section{Authors' contributions}

DZ carried out studies on the linear algebra and matrix theory with applications, and drafted the manuscript. HL helped prove some lemmas and theorems. ZG read the manuscript carefully and gave valuable suggestions and comments. All authors read and approved the final manuscript.

\section{Competing interests}

The authors declare that they have no competing interests.

Received: 4 July 2011 Accepted: 12 March 2012 Published: 12 March 2012

\section{References}

1. Zhang, F: Matrix Theory: Basic Results and Techniques. Springer, New York (1999)

2. Liu, ZY: Some properties of centrosymmetric matrices. Appl Math Comput. 141, 17-26 (2002)

3. Ulukök, Z, Türkmen, R: On some matrix trace inequalities. J Inequal Appl 2010 (2010). Article ID 201486

4. Li, HY, Gao, ZS, Zhao, D: A note on Frobenius conditional number with positive definite matrices. J Inequal Appl.2011

doi:10.1186/1029-242X-2012-62

Cite this article as: Zhao et al:: Trace inequalities for positive semidefinite matrices with centrosymmetric structure. Journal of Inequalities and Applications 2012 2012:62.

\section{Submit your manuscript to a SpringerOpen ${ }^{\circ}$ journal and benefit from:}

Convenient online submission

Rigorous peer review

- Immediate publication on acceptance

- Open access: articles freely available online

- High visibility within the field

- Retaining the copyright to your article

Submit your next manuscript at $\boldsymbol{s p r i n g e r o p e n . c o m ~}$ 